\title{
Prevalence and Risk Factors of Abusive Behaviors in the Caregivers of People with Dementia in Korea
}

\author{
Taehyun Kim¹, Hyeon Jeong², Ji Won Han¹, Kyung Phil Kwak³, Bong-Jo Kim4, Shin-Kyeom Kim5, \\ Jeong Lan Kim ${ }^{6}$, Tae Hui Kim ${ }^{7}$, Seung-Ho Ryu ${ }^{8}$, Seok Woo Moon ${ }^{9}$, Jae Young Park', Joon Hyuk Park ${ }^{10}$, \\ Jae Nam Bae ${ }^{11}$, Jong Chul Youn ${ }^{12}$, Dong Young Lee ${ }^{13,14}$, Dong Woo Lee ${ }^{15}$, Seok Bum Lee ${ }^{16}$, \\ Jung Jae Lee ${ }^{16}$, Jin Hyeong Jhoo ${ }^{17}$, Jungwon Lee ${ }^{1}$, and Ki Woong Kim ${ }^{1,14,18,19 凶}$ \\ ${ }^{1}$ Department of Neuropsychiatry, Seoul National University Bundang Hospital, Seongnam, Republic of Korea \\ ${ }^{2}$ Department of Psychiatry, Mirae Hospital, Gimje, Republic of Korea \\ ${ }^{3}$ Department of Psychiatry, Dongguk Medical Center, Gyeongju, Republic of Korea \\ ${ }^{4}$ Department of Psychiatry, Gyeongsang National University School of Medicine, Jinju, Republic of Korea \\ ${ }^{5}$ Department of Neuropsychiatry, Soonchunhyang University Bucheon Hospital, Bucheon, Republic of Korea \\ ${ }^{6}$ Department of Psychiatry, Chungnam National University Hospital, Daejeon, Republic of Korea \\ ${ }^{7}$ Department of Psychiatry, Yonsei University Wonju Severance Christian Hospital, Wonju, Republic of Korea \\ ${ }^{8}$ Department of Psychiatry, School of Medicine, Konkuk University, Konkuk University Medical Center, Seoul, Republic of Korea \\ ${ }^{9}$ Department of Psychiatry, School of Medicine, Konkuk University, Konkuk University Chungju Hospital, Chungju, Republic of Korea \\ ${ }^{10}$ Department of Neuropsychiatry, Jeju National University Hospital, Jeju, Republic of Korea \\ ${ }^{11}$ Department of Psychiatry, Inha University Hospital, Incheon, Republic of Korea \\ ${ }^{12}$ Department of Neuropsychiatry, Kyunggi Provincial Hospital for the Elderly, Yongin, Republic of Korea \\ ${ }^{13}$ Department of Neuropsychiatry, Seoul National University Hospital, Seoul, Republic of Korea \\ ${ }^{14}$ Department of Psychiatry, Seoul National University College of Medicine, Seoul, Republic of Korea \\ ${ }^{15}$ Department of Neuropsychiatry, Inje University Sanggye Paik Hospital, Seoul, Republic of Korea \\ ${ }^{16}$ Department of Psychiatry, Dankook University Hospital, Cheonan, Republic of Korea \\ ${ }^{17}$ Department of Psychiatry, Kangwon National University School of Medicine, Chuncheon, Republic of Korea \\ ${ }^{18}$ Department of Brain and Cognitive Science, Seoul National University College of Natural Sciences, Seoul, Republic of Korea \\ ${ }^{19}$ National Institute of Dementia, Seongnam, Republic of Korea
}

Objective We investigated the prevalence and risk factors of physically abusive behaviors $(\mathrm{PhAB})$ and psychologically abusive behaviors (PsAB) towards people with dementia (PWD) in family caregivers, and compared their prevalences between East Asian and Western countries. Methods We estimated the prevalence and risk factors of PhAB and PsAB in 467 Korean pairs of community-dwelling PWD and their primary family caregivers. We evaluated abusive behaviors using the Modified Conflict Tactics Scale. In addition, we compared the prevalence of abusive behaviors between Asian and Western countries through a meta-analysis on 12 studies including the current one.

Results More than a half of the caregivers reported PsAB and about one out of seven caregivers admitted PhAB within past three months. PsAB and PhAB were slightly more prevalent in East Asian countries than in Western countries. Non-Alzheimer type and moderate to severe behavioral and psychological symptoms of dementia were associated with the risk of PhAB but not with the risk of $\mathrm{PsAB}$. Severe care burden and low income were associated with the risk of $\mathrm{PhAB}$ and PsAB.

Conclusion $\mathrm{PhAB}$ and PsAB were as prevalent in the family caregivers of PWD in Asian countries including Korea as in Western countries. Prevention strategies should be implemented according to the type of abusive behaviors. Psychiatry Investig 2018;15(7):677-686

Key Words Abuse, dementia, Prevalence, Risk factors.

\footnotetext{
Received: January 7, 2018 Revised: March 27, 2018 Accepted: April 23, 2018

$\triangle$ Correspondence: Ki Woong Kim, MD, PhD

Department of Neuropsychiatry, Seoul National University College of Medicine and Seoul National University Bundang Hospital, 166 Gumi-ro, Bundang-gu, Seongnam 13620, Republic of Korea

Tel: +82-31-787-7432, Fax: +82-31-787-4058, E-mail: kwkimmd@snu.ac.kr

(a) This is an Open Access article distributed under the terms of the Creative Commons Attribution Non-Commercial License (http://creativecommons.org/licenses/by-nc/4.0) which permits unrestricted non-commercial use, distribution, and reproduction in any medium, provided the original work is properly cited.
} 


\section{INTRODUCTION}

Along with the rapid increase of people with dementia (PWD), ${ }^{1,2}$ the high prevalence of abusive behaviors toward PWD caused widespread public alarm in South Korea (hereafter, Korea). In the first Nationwide Survey on Dementia Epidemiology of Korea, which was conducted in 2008, 54\% of family caregivers of community-dwelling PWD admitted to one or more abusive behaviors within the prior month, ${ }^{3}$ which was approximately nine times higher, than those caring for the healthy older population $(6.3 \%) .{ }^{4} \mathrm{PWD}$ are at a greater risk for abuse, with a reported prevalence of abuse of 27.9-62.3\%, compared with the elderly with normal cognition where the prevalence was $3.2-27.5 \%{ }^{5-19}$ Furthermore, older people, particularly PWD, can be more vulnerable to the impact of abuse than younger adults due to their limited physical and psychological strength, and resilience. ${ }^{20,21}$

The risk of abuse in PWD is determined by complex interactions among the characteristics of PWD [i.e., higher morbidity, severity of cognitive impairments, and behavioral and psychological symptoms of dementia (BPSD)], their caregivers (i.e., cultural background, psychological morbidity, dysfunctional coping strategy, and worse relationship with the care recipient) and environment (i.e., long caring time, high perceived burden, and poor social support). 12,16,17,22,23 In this respect, abuse of PWD may be less prevalent in the East Asian countries under Confucian culture, such as Korea, China and Japan, where respect towards elders and filial devotion are long-held traditions and still important social virtues. However, no study has directly compared the prevalence of abusive behavior towards PWD between Asian and Western countries. Furthermore, most previous studies on abuse of PWD were conducted on small samples, ${ }^{5-9,11-19}$ some did not consider the subtypes of abuse $e^{9-11,19}$ or kinship of caregivers, ${ }^{7,14-17,19}$ and some did not differentiate professional caregivers and family caregivers. ${ }^{7,15,17}$

In the current study, as a part of the Nationwide Survey on Dementia Care in Korea (NaSDeCK), ${ }^{3}$ we separately investigated the prevalence and risk factors of physically and psychologically abusive behaviors in a large nationwide sample of community-dwelling PWD and their family caregivers. In addition, we compared the prevalence of abusive behaviors between Asian countries under Confucian culture and Western countries, using a meta-analysis on 11 previous studies and the current study.

\section{METHODS}

\section{Study population}

The NaSDeCK was a nationwide multi-centered study on dementia care in Korea, which was conducted from January 2011 to December 2011. Among the 731 pairs of dementia patients and their caregivers who participated in the NaSDeCK, 467 pairs of community-dwelling PWD and their family caregivers (i.e., spouses or children) were included in the study cohort. All family caregivers met the PWD at least once a week $(n=221)$ or lived with them $(n=246)$. All participants provided written informed consent, either by themselves or their legal guardians. The Institutional Review Board of Seoul National University Bundang Hospital approved this study (IRB No: B-1008/110-001).

\section{Assessments}

A face-to-face standardized diagnostic interview and physical and neurological examinations were administered to each subject with dementia, using the Korean version of the Consortium to Establish a Registry for Alzheimer's Disease Assessment Packet (CERAD-K) Clinical Assessment Battery (CERAD-K-C) ${ }^{24}$ Geriatric psychiatrists, who were certified to administer the CERAD-K-C, conducted these assessments. The CERAD-K Neuropsychological Assessment Battery (CERAD-K-N) $)^{24,25}$ was also administered by neuropsychologists or trained research nurses. The CERAD-K-N consists of nine neuropsychological tests as follows: Verbal Fluency Test, 15-item Boston Naming Test, Mini Mental Status Examination (MMSE), Word List Memory Test, Constructional Praxis Test, Word List Recall Test, Word List Recognition Test, Constructional Recall Test, and Trail Making Test. Brain computed tomography or magnetic resonance imaging, laboratory tests, including complete blood cell count, chemistry profile, serological test for syphilis and HIV, thyroid function test, vitamin B12/folate, and echocardiogram and chest X-ray scan were conducted for participants who were diagnosed with dementia, in order to determine the subtype of dementia.

Dementia was first defined according to the Diagnostic and Statistical Manual of Mental Disorders, Fourth Edition (DSM-IV) diagnostic criteria. ${ }^{26}$ For the participants who met the DSM-IV diagnostic features of dementia, subtypes of dementia were further determined as follows: Alzheimer's disease (AD) according to the criteria of the National Institute of Neurological and Communicative Disorders and Stroke and the Alzheimer's Disease and Related Disorders Association (NINCDS-ADRDA), ${ }^{2}$ vascular dementia (VD) according to the criteria of the National Institute of Neurological Disorders and Stroke-Association Internationale pour la Recherche et l'Enseignement en Neurosciences (NINDS-AIREN), ${ }^{27}$ Lewy body dementia and Parkinson's disease with dementia according to the consensus guideline proposed by McKeith et al. ${ }^{28}$ frontotemporal dementia according to the 
Table 1. Characteristics of 467 pairs of the people with dementia (PWD) and their caregivers

\begin{tabular}{|c|c|c|}
\hline & PWD & Caregivers \\
\hline Age (years) & $76.97 \pm 7.57$ & $57.77 \pm 13.99$ \\
\hline Sex (female, \%) & 63.81 & 66.60 \\
\hline Education (years) & $6.64 \pm 5.43$ & $11.89 \pm 4.70$ \\
\hline Type of dementia (Alzheimer's type, \%) & 72.16 & - \\
\hline Clinical dementia rating (\%) & & - \\
\hline 0.5 (very mild) & 28.27 & - \\
\hline 1 (mild) & 49.25 & - \\
\hline 2 (moderate) & 17.34 & - \\
\hline 3 (severe) & 5.14 & - \\
\hline Mini Mental Status Examination (points) & $16.16 \pm 5.28$ & - \\
\hline Disability Assessment for Dementia (points) & $0.63 \pm 0.30$ & - \\
\hline Duration of illness (years) & $4.13 \pm 3.18$ & - \\
\hline Neuropsychiatric Inventory (points) & $12.40 \pm 15.75$ & - \\
\hline Moderate to severe BPSD (\%)* & 65.52 & - \\
\hline Cumulative Illness Rating Scale (points) & $6.16 \pm 3.37$ & - \\
\hline Disadvantaged income $(<1,763$ USD/month, \%) & - & 42.83 \\
\hline Kinship (spouses, \%) & - & 38.54 \\
\hline Duration of care before dementia diagnosis (years) & - & $17.36 \pm 20.32$ \\
\hline Duration of care after dementia diagnosis (years) & - & $3.37 \pm 2.96$ \\
\hline Daily caring time (hours/day) & - & $4.67 \pm 4.54$ \\
\hline Dementia Attitude Scale (points) & - & $79.69 \pm 15.82$ \\
\hline Social comfort score (points) & - & $37.48 \pm 10.10$ \\
\hline Knowledge score (points) & - & $42.21 \pm 9.72$ \\
\hline Zarit Burden Interview Short Form (points) & - & $22.01 \pm 9.76$ \\
\hline Severe perceived care burden ( $\geq 12$ points, $\%$ ) & - & 85.44 \\
\hline Living area (urban, \%) & - & 84.15 \\
\hline National Longterm Care Service (\%) & - & 21.97 \\
\hline
\end{tabular}

*3 or over on depression subscale (severity $\times$ frequency), 5 or over on anxiety, 2 or over on apathy, 5 or over on irritability, night-time and appetite/eating, or 1 or over on delusion, hallucination, agitation, euphoria, disinhibition and aberrant motor behavior in the Neuropsychiatric Inventory. ${ }^{33}$ BPSD: behavioral and psychological symptoms of dementia

Lund-Manchester consensus diagnostic criteria, ${ }^{29}$ and alcohol-related dementia according to the diagnostic criteria proposed by Oslin et al..$^{30}$

We evaluated the abusive behavior of the caregivers using the Modified Conflict Tactics Scale (MCTS) ${ }^{31}$ The MCTS consists of five questions on psychological abuse (i.e., screamed or shouted; used a harsh tone of voice, insulted, sworn at, or called them names; threatened to send them to a care home; stop taking care of or abandoned them; and threatened to use physical force on them) and five on physical abuse (i.e., had been afraid they might hit or hurt them; had withheld food; had hit or slapped them; had shaken; and had handled them roughly in other ways). We asked caregivers how often within the prior 3 months they had acted in each of five psychologically and five physically abusive ways toward the
PWD, and scored them using a Likert scale from 0 (never) to 4 (all the time). We avoided the mention of the word 'abuse' when we applied the MCTS to the caregivers to reduce their defensive attitude. A score of 2 or over (sometimes) for any question was regarded as significant abuse.

We evaluated the characteristics of PWD, their caregivers, and environment that were potentially associated with the risk of abusive behavior, using the instruments listed in Table 1 . Patient factors included age, education, sex, type of dementia, severity of dementia measured by the Clinical Dementia Rating scale, global cognition measured by MMSE, duration of illness, burden of comorbid diseases measured by the $\mathrm{Cu}-$ mulative Illness Rating Scale, ${ }^{32}$ and severity of BPSD measured by the Neuropsychiatric Inventory. ${ }^{33}$ We defined moderate to severe BPSD as 3 points or over on the depression 
subscale (severity $\times$ frequency), 5 points or over on anxiety, 2 points or over on apathy, 5 points or over on irritability, night-time and appetite/eating, or 1 point or over on delusion, hallucination, agitation, euphoria, disinhibition, and aberrant motor behavior on the Neuropsychiatric Inventory. ${ }^{33}$ Caregiver factors included age, education, sex, kinship, monthly income, duration of care after dementia diagnosis (DOCAD), duration of care before dementia diagnosis, daily caring time, severity of perceived care burden measured by the Zarit Burden Interview Short Form (sZBI), ${ }^{34}$ and attitude to and knowledge of dementia measured by Dementia Attitude Scale (DAS). ${ }^{35}$ We defined low income as a monthly household income below 1,763 USD, poor attitude to dementia as the 25th percentile or lower on the DAS Stability subscore, poor knowledge of dementia as the 25th percentile or below on the DAS Knowledge subscore, and severe care burden as 12 points or over on the sZBI. ${ }^{34}$ Environmental factors included living areas (rural versus urban) and the use of the National Longterm Care Insurance (NLCI).

\section{Meta-analysis on previous literatures}

This meta-analysis included the studies that enrolled people diagnosed with any type of dementia according to the DSM-IV diagnostic criteria and their caregivers, and employed cross-sectional designs and reported the prevalence estimates of abusive behaviors toward PWD in family caregivers.

One reviewer (Kim T) searched the MEDLINE ${ }^{\circledR}$ and selected the publications written in English only through three phases; identified the potential papers for abstract retrieval and eliminated any obviously irrelevant ones (initial screening), identified the potential papers for full text retrieval by screening their abstracts (secondary screening), and identified the papers that should be included in the current metaanalysis by reading the full text (tertiary screening). One reviewer (Kim T) collected the data and conducted the metaanalysis. We used Boolean operation ('OR') for sensitive search. The searching strategies were as following;

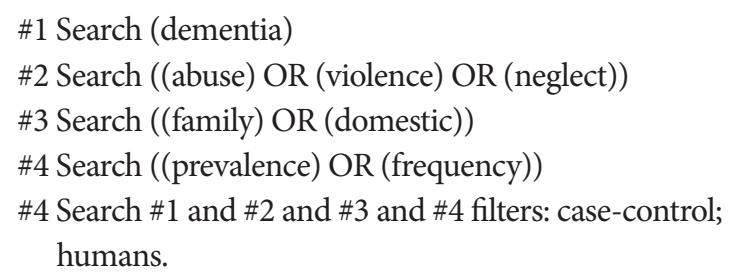

Finally, we included 12 studies in the current meta-analysis (Supplement Table 1 in the online-only Data Supplement).

\section{Statistical analysis}

We summarized the characteristics of subjects using counts, percentages, or mean \pm standard deviation (SD). We estimated unweighted frequencies with $95 \%$ confidence intervals (CI) of overall abusive behaviors, psychologically abusive behaviors alone (PsAB), and physically abusive behaviors with or without PsAB (PhAB). We also performed a metaanalysis on 11 previous studies that reported unweighted frequencies of abusive behaviors in caregivers of $\mathrm{PWD}^{5-9,11-17}$ and the current study, to compare the frequencies of abusive behaviors in the caregivers of PWD between Asian countries and Western countries. Substantial heterogeneity was analyzed in frequency estimates for abusive behaviors among the studies using Cochran's $\mathrm{Q}$ (reported with $\chi^{2}$ and $\mathrm{p}$ ) and the $\mathrm{I}^{2}$ statistic. The forest plot was used to present the extent of heterogeneity among studies. The influence of country distribution (Asian and Western countries) controlled the study design (cross-sectional and longitudinal studies) on estimated frequencies was investigated using meta-regression and suggested as odds ratios.

We compared the risk of abusive behaviors between spouse caregivers and adult child caregivers using univariate logistic regression analyses. We also examined PWD, caregiver, and environmental factors that were associated with the risk of $\mathrm{PsAB}$ and $\mathrm{PhAB}$, using hierarchical multivariate multinomial logistic regression analyses. Data were analyzed using STATA 14.0 (Stata Corp., College Station, TX, USA).

\section{RESULTS}

\section{Characteristics of people with dementia and their caregivers}

Approximately three quarters of PWD were diagnosed as $\mathrm{AD}$, and approximately two thirds had moderate to severe BPSD. The mean duration of illness was approximately 4 years. Approximately two thirds of the caregivers were women, and four out of ten caregivers were spouses of PWD. On average, caregivers had supported their family member with dementia for approximately 20 years since they had been cognitively normal, and for approximately 3.4 years after they had become demented. Caregivers took care of their family member, on average, for approximately $5 \mathrm{~h} /$ day. More than $80 \%$ of caregivers reported a severe care burden (i.e., 12 or higher on sZBI), but only approximately $20 \%$ used the services of the NLCI.

\section{Frequencies of abusive behaviors (Table 2, Figure 1)}

More than half of the caregivers reported one or more abusive behaviors toward their family member with dementia within the prior 3 months; more than half of the caregivers reported PsAB, while approximately one out of seven caregivers admitted PhAB. Over $97 \%$ of family caregivers who showed 
Table 2. Prevalence* of abusive behaviors in family caregivers of people with dementia in Korea

\begin{tabular}{|c|c|c|c|c|c|}
\hline & \multicolumn{3}{|c|}{ Kinship } & \multicolumn{2}{|l|}{ Statistics } \\
\hline & All $(\mathrm{N}=467)$ & Spouses $(\mathrm{N}=180)$ & Children $(\mathrm{N}=287)$ & $\mathrm{OR}(95 \% \mathrm{CI})^{\dagger}$ & $\mathrm{p}$ \\
\hline Any verbal abuse & $51.6(47.1-56.1)$ & $57.8(50.4-64.8)$ & $47.7(42.0-53.6)$ & $0.67(0.46-0.97)$ & 0.034 \\
\hline Screamed and yelled at & $49.7(45.1-54.2)$ & $54.4(47.1-61.6)$ & $46.7(41.0-52.5)$ & $0.73(0.50-1.07)$ & 0.103 \\
\hline Used harsh tone of voice, insulted, swore at & $21.6(18.1-25.6)$ & $28.3(22.2-35.4)$ & $17.4(13.4-22.3)$ & $0.53(0.34-0.83)$ & 0.006 \\
\hline Threatened to send to nursing home & $10.1(7.6-13.2)$ & $11.1(7.3-16.7)$ & $9.4(6.5-13.4)$ & $0.83(0.45-1.53)$ & 0.554 \\
\hline Threatened to stop taking care of & $3.0(1.8-0.5)$ & $4.4(2.2-8.7)$ & $2.1(0.9-4.6)$ & $0.46(0.16-1.35)$ & 0.153 \\
\hline Threatened to use physical force & $2.6(1.5-4.5)$ & $3.3(1.5-7.3)$ & $2.1(0.9-4.6)$ & $0.62(0.20-1.95)$ & 0.415 \\
\hline Any physical abuse & $13.9(11.1-17.4)$ & $18.3(13.3-24.7)$ & $11.1(8.0-15.4)$ & $0.56(0.33-0.95)$ & 0.031 \\
\hline Afraid caregiver might hit or try to hurt & $7.9(5.8-10.8)$ & $9.4(5.9-14.7)$ & $7.0(4.5-10.6)$ & $0.72(0.37-1.41)$ & 0.339 \\
\hline Withheld food & $0.9(0.3-2.3)$ & $0.6(0.1-3.9)$ & $1.0(0.3-3.2)$ & $1.89(0.20-18.32)$ & 0.565 \\
\hline Hit or slapped & $2.1(1.2-3.9)$ & $3.3(1.5-7.3)$ & $1.4(0.5-3.7)$ & $0.41(0.11-1.47)$ & 0.166 \\
\hline Shook & $7.1(5.1-9.8)$ & $8.9(5.5-14.1)$ & $5.9(3.7-9.3)$ & $0.65(0.32-1.31)$ & 0.229 \\
\hline Handled roughly in other ways & $5.8(4.0-8.3)$ & $7.2(4.2-12.1)$ & $4.9(2.9-8.1)$ & $0.66(0.30-1.44)$ & 0.296 \\
\hline Any abuse & $52.0(47.5-56.6)$ & $57.8(50.4-64.8)$ & $48.4(42.7-54.2)$ & $0.69(0.47-1.00)$ & 0.049 \\
\hline
\end{tabular}

${ }^{*}$ prevalence estimates with $95 \%$ confidence intervals (CIs), $\%,{ }^{\dagger}$ odds ratio (OR) with CIs of children for abusive behaviors compared to spouses

physically abusive behaviors also exhibited psychologically abusive behavior. Screaming and yelling were the most common type of PsAB (49.7\%), while being afraid that caregiver might hit or try to hurt them (7.9\%) and shaking $(7.1 \%)$ were the two most common forms of PhAB. Abusive behavior was more prevalent in spouses than in children, when we analyzed PsAB and PhAB separately. Compared to spouses, adult children were less likely to show both PsAB and PhAB.

In our meta-analysis, the frequencies of PsAB and $\mathrm{PhAB}$ were estimated to be $41.5 \%$ ( $95 \% \mathrm{CI}=34.7-48.3 \%$ ) and $10.0 \%$ (95\% CI=7.0-12.9\%), respectively (Figure 1). Q statistics and $\mathrm{I}^{2}$ analysis for heterogeneity were $76.44 \%$ and $85.61 \%$ for overall abusive behaviors; $82.28 \%$ and $87.85 \%$ for PsAB; and $73.74 \%$ and $83.73 \%$ for $\mathrm{PhAB}$, respectively. In the multivariate meta-regression adjusting study designs (cross-sectional versus prospective), $\mathrm{Ps} \mathrm{AB}$ and $\mathrm{PhAB}$ were slightly more prevalent in East Asian countries than in Western countries [odd ratio (OR) $=1.201,95 \% \mathrm{CI}=1.012-1.426, \mathrm{p}=0.039$ for PsAB; $\mathrm{OR}=1.070,95 \% \mathrm{CI}=0.988-1.160, \mathrm{p}=0.087$ for $\mathrm{PhAB}]$.

\section{Factors associated with abusive behavior (Table 3 )}

Among patient factors the risk of $\mathrm{PhAB}$ was associated with non-Alzheimer type $(\mathrm{OR}=2.04,95 \% \mathrm{CI}=1.04-4.00, \mathrm{p}=0.037)$ and moderate-to-severe BPSD $(\mathrm{OR}=2.33,95 \% \mathrm{CI}=1.12-4.84$, $\mathrm{p}=0.023)$, while among caregiver factors it was associated with low income $(\mathrm{OR}=2.84,95 \% \mathrm{CI}=1.36-5.93, \mathrm{p}=0.005)$, poor knowledge about dementia $(\mathrm{OR}=2.07,95 \% \mathrm{CI}=1.04-$ $4.15, \mathrm{p}=0.38)$, and severe perceived care burden $(\mathrm{OR}=6.24$, $95 \% \mathrm{CI}=1.69-22.97, \mathrm{p}=0.006$ ). The risk of PsAB was associated with severe dementia $(\mathrm{OR}=0.27,95 \% \mathrm{CI}=0.08-0.88, \mathrm{p}=$
$0.031)$ among patient factors, and with low income $(\mathrm{OR}=1.83$, 95\% $\mathrm{CI}=1.09-3.09, \mathrm{p}=0.022)$, longer DOCAD (OR $=1.16,95 \%$ $\mathrm{CI}=1.00-1.33, \mathrm{p}=0.039)$, and severe care burden $(\mathrm{OR}=4.45$, 95\% CI=2.15-9.19, $\mathrm{p}<0.001)$ among caregiver factors. A higher level of education of PWD was associated with the reduced risk of PsAB (OR=0.94, 95\% CI=0.89-0.99, $\mathrm{p}=0.046$ ). The risks of $\mathrm{Ps} \mathrm{AB}$ and $\mathrm{PhAB}$ were not associated with environmental factors.

\section{DISCUSSION}

We found that abusive behavior was quite prevalent in family caregivers of community-dwelling PWD in Korea, with one out of two caregivers and one out of seven caregiver admitting admitted $\mathrm{Ps} \mathrm{AB}$ and $\mathrm{PhAB}$, respectively, within the prior 3 months. In many East Asian countries, including Korea and China, filial piety, i.e., supporting and serving one's parents, is regarded as one's natural duty. Filial piety dictates obligatory roles and responsibilities of children in caregiving for aging parents. In return, parents are expected to contribute to the harmony of family and society with their guidance and wisdom. ${ }^{36}$ However, against our expectation, that this traditional virtue of filial piety may be protective against abusive behaviors toward PWD in East Asian countries under Confucian culture, our meta-analysis showed that PsAB were more prevalent and $\mathrm{PhAB}$ were comparably prevalent in East Asian countries, compared with Western countries. Although the frequencies of both PsAB and PhAB were less common in child caregivers, compared with spouse caregivers, kinship was not associated with the risk of PsAB and 


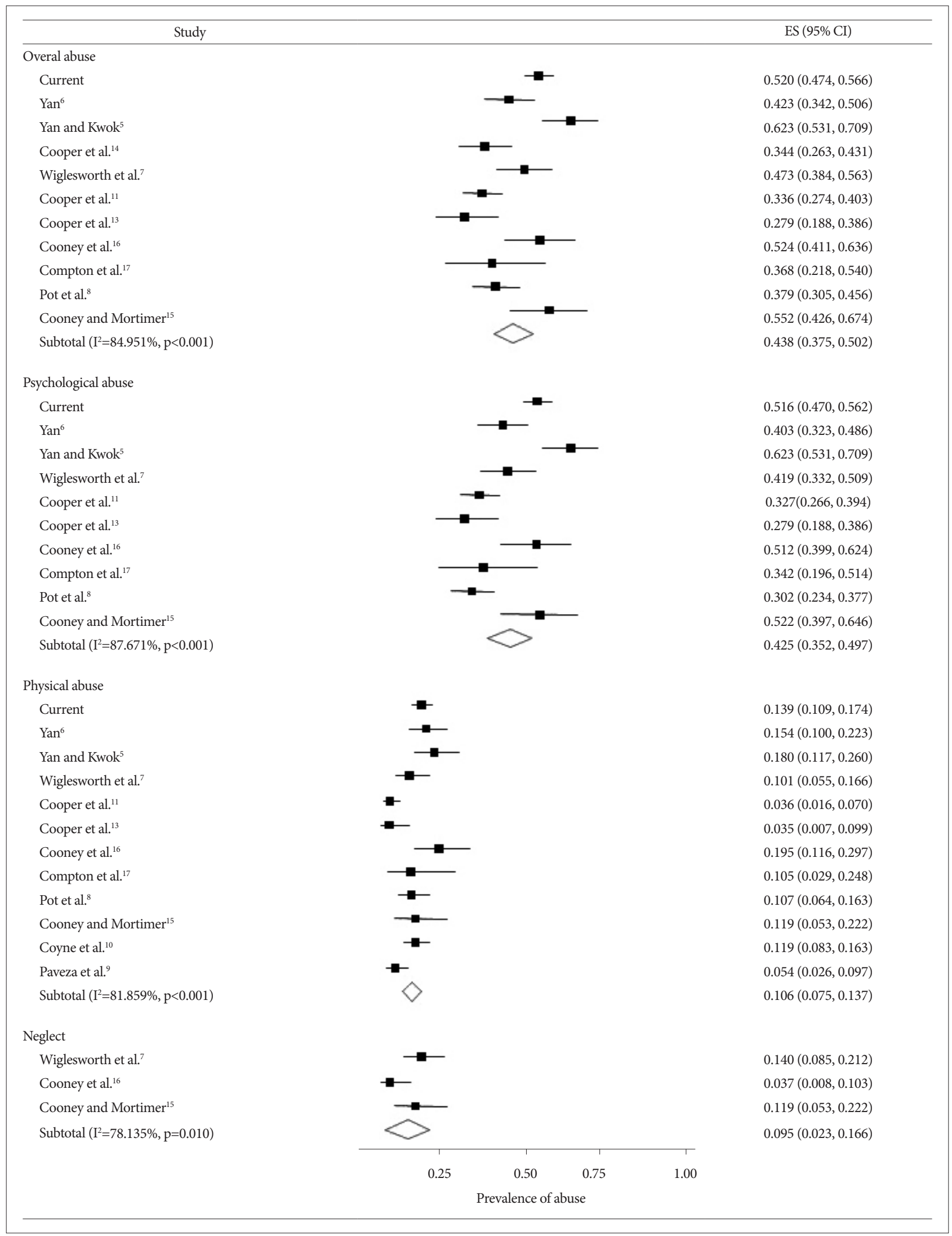

Figure 1. Forest plot showing the proportion of caregivers' abusive behavior towards people with dementia. The plot also shows the subgroup analysis for overall abuse, psychological abuse, physical abuse, and neglect. 
T Kim et al.

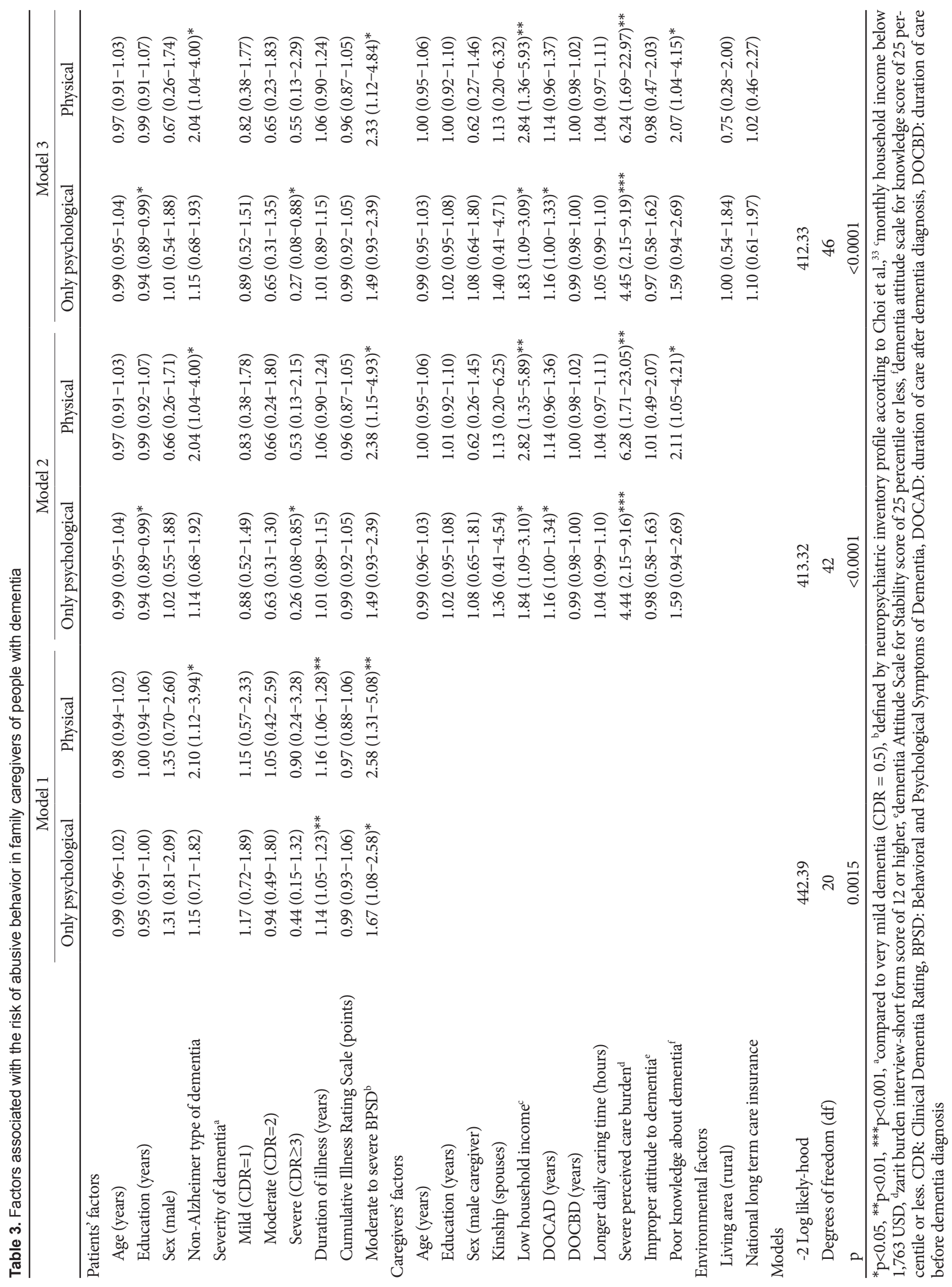


$\mathrm{PhAB}$ when other patient and caregiver factors were considered together. Rapid sociocultural transformations such as modernization, urbanization, and industrialization, which have occurred in the past several decades in Korea may have contributed to the high risk for abusive behavior towards PWD within families, by altering family structures and challenging traditional family values. The younger generation have become less adherent to traditional Confucian principles of filial piety; older generations are no longer guaranteed prestige and care in the family. ${ }^{37}$ In 1998, 89.9\% of Korean people thought that they should support and serve their parents. However, in 2016, the proportion dropped considerably to $36.6 \% .{ }^{38}$ Factors that were not surveyed in the present study, such as current or previous relationship between PWD and their children, could have also contributed to the prevalent abusive behavior of adult child caregivers. ${ }^{15-17}$

To reduce the incidence and impact of abusive behavior towards PWD, it is important to define and monitor a highrisk group for abusive behavior, and to provide them with early intervention programs. However, abusive behaviors are underreported due to high cultural sensitivity, a low level of awareness, reluctance to reveal the case in order to maintain family harmony and honor, and the perception that elder abuse is a private family matter in East Asian countries. ${ }^{39} \mathrm{~Pa}-$ tient and caregiver factors that were associated with the risk of abusive behaviors toward PWD in the present study may help us define PWD who are at risk of domestic abuse and provide them with contextually appropriate prevention and intervention programs.

Among caregiver factors, severe perceived care burden was the most strongly associated with the approximate four-fold increase of PsAB and six-fold increase of PhAB. The association between perceived care burden and the risk of abuse has been consistently replicated in both Western and East Asian countries. ${ }^{5,7,11,13,18,19}$ In addition, low household income was associated with the risk of both $\mathrm{PhAB}$ and $\mathrm{PsAB}$, poor knowledge about dementia was associated with the risk of $\mathrm{PhAB}$, and longer DOCAD was associated with the risk of PsAB. Although three previous studies reported that household income was not associated with the risk of abusive behaviors, the average household income of the participants in those studies were higher than that of the participants in the current study. ${ }^{5-7}$ Therefore, the assessment of perceived care burden of caregivers may be the first step in detecting a highrisk group for abusive behavior. Among patient factors, nonAlzheimer type dementia and moderate to severe BPSD were associated with the two-fold increase of $\mathrm{PhAB}$. This is the first study to investigate the differential impact of dementia subtypes on the risk of abusive behaviors. Compared with $\mathrm{AD}$, non-Alzheimer's type dementia, such as Lewy body de- mentia or frontotemporal dementia, may have more BPSD from an earlier stage, resulting in greater perceived care burden. ${ }^{40,41}$ PsAB became less prevalent in severe stages of dementia, but $\mathrm{PhAB}$ did not, which could be attributable to reduced communication between PWD and their caregivers, due to a loss of the patients' cognitive and physical abilities to communicate at late stages of the disease. ${ }^{16}$

In summary, the risk of $\mathrm{PhAB}$ may be attributed to an increase in perceived care burden, due to the characteristics of dementia, such as type of dementia or presence of BPSD, and/or the lack of proper knowledge about dementia. In contrast, $\mathrm{PhAB}$ could be attributed to an increased in perceived care burden due to prolonged duration of dementia care. Therefore, we could reduce the risk of PhAB towards PWD by family caregivers if PWD are provided with proper intervention for BPSD as early as possible, and family caregivers are adequately educated about dementia. These services should more readily available when the patient has non-Alzheimer type dementia and their caregivers' income is disadvantaged to actively reduce the risk of $\mathrm{PhAB}$. In contrast, the risk of PsAB can be reduced by easing caregiver burden, specifically the total amount of required dementia care, by providing tailored tangible support. In the current study cohort, $21.97 \%$ of PWD were using home care services provided by the NLCI, which did not seem effective at reducing the PhAB and PsAB in the current study. This indicates that services provided by Korean NLCI may be either insufficient or ineffective in reducing care burden in its current form. In many previous studies, various psychosocial intervention programs were only weakly effective in relieving psychological distress of caregivers of PWD. ${ }^{42-45}$ Respite care, a type of tangible support, was not effective in reducing psychological distress of caregivers of PWD either. ${ }^{46}$ However, if we tailored such services differentially, according to the causes of perceived care burden, we may noticeably enhance their efficacy in reducing care burden, and thus, the risk of abusive behavior.

A few limitations of the current study must be noted in order to correctly interpret its results. First, since this was a cross-sectional study, causal relationships cannot be inferred. Second, the frequency of abusive behavior might have been underestimated, since the family caregivers self-reported their abusive behavior using the administered questionnaire. Third, we did not evaluate other types of abusive behavior, such as neglect, financial exploitation, and sexual abuse.

In conclusion, both $\mathrm{PhAB}$ and $\mathrm{Ps} \mathrm{AB}$ were prevalent in family caregivers of PWD in the countries under Confucian culture. Prevention strategies should be tailored individually, in accordance with differential risk factors, in order to reduce $\mathrm{PhAB}$ and PsAB of PWD. 


\section{Supplementary Materials}

The online-only Data Supplement is available with this article https://doi.org/10.30773/pi.2018.04.23.

\section{Acknowledgments}

This study was supported by a grant from the Korean Health Technology R\&D Project, Ministry of Health and Welfare, Republic of Korea [Grant No. HI09C1379 (A092077)].

\section{REFERENCES}

1. Kim KW, Park JH, Kim MH, Kim MD, Kim BJ, Kim SK, et al. A nationwide survey on the prevalence of dementia and mild cognitive impairment in South Korea. J Alzheimers Dis 2011;23:281-291.

2. McKhann G, Drachman D, Folstein M, Katzman R, Price D, Stadlan EM. Clinical diagnosis of Alzheimer's disease: report of the NINCDSADRDA Work Group under the auspices of Department of Health and Human Services Task Force on Alzheimer's Disease. Neurology 1984;34:939-944.

3. The National Survey of Dementia Care in Korea (NaSDeCK): 111352000-000576-15. Sejong: Ministry of Health and Welfare; 2011.

4. Oh J, Kim HS, Martins D, Kim H. A study of elder abuse in Korea. Int J Nurs Stud 2006;43:203-214.

5. Yan E, Kwok T. Abuse of older Chinese with dementia by family caregivers: an inquiry into the role of caregiver burden. Int J Geriatr Psychiatry 2011;26:527-535.

6. Yan E. Abuse of older persons with dementia by family caregivers: results of a 6-month prospective study in Hong Kong. Int J Geriatr Psychiatry 2014;29:1018-1027.

7. Wiglesworth A, Mosqueda L, Mulnard R, Liao S, Gibbs L, Fitzgerald W. Screening for abuse and neglect of people with dementia. J Am Geriatr Soc 2010;58:493-500.

8. Pot AM, van Dyck R, Jonker C, Deeg DJ. Verbal and physical aggression against demented elderly by informal caregivers in The Netherlands. Soc Psychiatry Psychiatr Epidemiol 1996;31:156-162.

9. Paveza GJ, Cohen D, Eisdorfer C, Freels S, Semla T, Ashford JW, et al. Severe family violence and Alzheimer's disease: prevalence and risk factors. Gerontologist 1992;32:493-497.

10. Coyne AC, Reichman WE, Berbig LJ. The relationship between dementia and elder abuse. Am J Psychiatry 1993;150:643-646.

11. Cooper C, Selwood A, Blanchard M, Walker Z, Blizard R, Livingston G. The determinants of family carers' abusive behaviour to people with dementia: results of the CARD study. J Affect Disord 2010;121:136-142.

12. Cooper C, Selwood A, Blanchard M, Walker Z, Blizard R, Livingston G. Abuse of people with dementia by family carers: representative cross sectional survey. BMJ 2009;338:b155.

13. Cooper C, Manela M, Katona C, Livingston G. Screening for elder abuse in dementia in the LASER-AD study: prevalence, correlates and validation of instruments. Int J Geriatr Psychiatry 2008;23:283-288.

14. Cooper C, Blanchard M, Selwood A, Walker Z, Livingston G. Family carers' distress and abusive behaviour: longitudinal study. Br J Psychiatry 2010;196:480-485.

15. Cooney C, Mortimer A. Elder abuse and dementia-a pilot study. Int J Soc Psychiatry 1995;41:276-283.

16. Cooney C, Howard R, Lawlor B. Abuse of vulnerable people with dementia by their carers: can we identify those most at risk? Int J Geriatr Psychiatry 2006;21:564-571.

17. Compton SA, Flanagan P, Gregg W. Elder abuse in people with dementia in Northern Ireland: Prevalence and predictors in cases referred to a psychiatry of old age service. Int J Geriatr Psychiatry 1997;12:632-635.

18. Cheng ST, Ip IN, Kwok T. Caregiver forgiveness is associated with less burden and potentially harmful behaviors. Aging Ment Health 2013;17: 930-934.
19. Camden A, Livingston G, Cooper C. Reasons why family members become carers and the outcome for the person with dementia: results from the CARD study. Int Psychogeriatr 2011;23:1442-1450.

20. Lachs MS, Williams CS, O'Brien S, Pillemer KA, Charlson ME. The mortality of elder mistreatment. JAMA 1998;280:428-432.

21. Dong X, Simon M, de Leon CM, Fulmer T, Beck T, Hebert L, et al. Elder Self-neglect and Abuse and Mortality Risk in a CommunityDwelling Population. JAMA 2009;302:517-526.

22. Shugarman LR, Fries BE, Wolf RS, Morris JN. Identifying older people at risk of abuse during routine screening practices. J Am Geriatr Soc 2003;51:24-31.

23. Dong X, Simon M, Rajan K, Evans DA. Association of Cognitive Function and Risk for Elder Abuse in a Community-Dwelling Population. Dement Geriatr Cogn Disord 2011;32:209-215.

24. Lee JH, Lee KU, Lee DY, Kim KW, Jhoo JH, Kim JH, et al. Development of the Korean version of the Consortium to Establish a Registry for Alzheimer's Disease Assessment Packet (CERAD-K): clinical and neuropsychological assessment batteries. J Gerontol B Psychol Sci Soc Sci 2002;57:P47-P53.

25. Lee DY, Lee KU, Lee JH, Kim KW, Jhoo JH, Kim SY, et al. A normative study of the CERAD neuropsychological assessment battery in the Korean elderly. J Int Neuropsychol Soc 2004;10:72-81.

26. American Psychiatric Association. Diagnostic and Statistical Manual of Mental Disorders: DSM-IV. Washington, DC: American Psychiatric Association; 1994.

27. Roman GC, Tatemichi TK, Erkinjuntti T, Cummings JL, Masdeu JC, Garcia JH, et al. Vascular dementia: diagnostic criteria for research studies. Report of the NINDS-AIREN International Workshop. Neurology 1993;43: 250-260.

28. McKeith IG, Galasko D, Kosaka K, Perry EK, Dickson DW, Hansen LA, et al. Consensus guidelines for the clinical and pathologic diagnosis of dementia with Lewy bodies (DLB): report of the consortium on DLB international workshop. Neurology 1996;47:1113-1124.

29. Neary D, Snowden JS, Gustafson L, Passant U, Stuss D, Black S, et al. Frontotemporal lobar degeneration: a consensus on clinical diagnostic criteria. Neurology 1998;51:1546-1554.

30. Oslin D, Atkinson RM, Smith DM, Hendrie H. Alcohol related dementia: proposed clinical criteria. Int J Geriatr Psychiatry 1998;13:203-212.

31. Beach SR, Schulz R, Williamson GM, Miller LS, Weiner MF, Lance CE. Risk factors for potentially harmful informal caregiver behavior. J Am Geriatr Soc 2005;53:255-261.

32. Miller MD, Paradis CF, Houck PR, Mazumdar S, Stack JA, Rifai AH, et al. Rating chronic medical illness burden in geropsychiatric practice and research: application of the Cumulative Illness Rating Scale. Psychiatry Res 1992;41:237-248.

33. Choi SH, Na DL, Kwon HM, Yoon SJ, Jeong JH, Ha CK. The Korean version of the neuropsychiatric inventory: a scoring tool for neuropsychiatric disturbance in dementia patients. J Korean Med Sci 2000;15: 609-615.

34. Higginson IJ, Gao W, Jackson D, Murray J, Harding R. Short-form Zarit Caregiver Burden Interviews were valid in advanced conditions. J Clin Epidemiol 2010;63:535-542.

35. O'Connor ML, McFadden SH. Development and psychometric validation of the Dementia Attitudes Scale. Int J Alzheimer Dis 2010;454218. doi:10.4061/2010/454218.

36. Chang ES, Dong X. Elder Abuse and Its Prevention: Workshop Summary. Washington, DC: National Academies Press (US); 2014.

37. Tsai JH. Meaning of filial piety in the Chinese parent-child relationship: implications for culturally competent health care. J Cult Divers 1999;6:26-34.

38. Kim Y. Family Support and Its Policy Implications in the Changing Social Environment: Health and Welfare Forum. Korea Institute for Health and Social Affairs 2017;252:6-28.

39. Dong X, Simon MA, Gorbien M, Percak J, Golden R. Loneliness in older chinese adults: a risk factor for elder mistreatment. J Am Geriatr 
Soc 2007;55:1831-1835.

40. Nyatsanza S, Shetty T, Gregory C, Lough S, Dawson K, Hodges J. A study of stereotypic behaviours in Alzheimer's disease and frontal and temporal variant frontotemporal dementia. J Neurol Neurosurg Psychiatry 2003;74:1398-1402.

41. Aharon-Peretz J, Kliot D, Tomer R. Behavioral differences between white matter lacunar dementia and Alzheimer's disease: a comparison on the neuropsychiatric inventory. Dement Geriatr Cogn Disord 2000;11:294-298.

42. Brodaty H, Green A, Koschera A. Meta-analysis of psychosocial interventions for caregivers of people with dementia. J Am Geriatr Soc 2003;51:657-664.

43. Bass DM, Clark PA, Looman WJ, McCarthy CA, Eckert S. The Cleve- land Alzheimer's managed care demonstration: outcomes after 12 months of implementation. Gerontologist 2003;43:73-85.

44. Done D, Thomas J. Training in communication skills for informal carers of people suffering from dementia: a cluster randomized clinical trial comparing a therapist led workshop and a booklet. Int J Geriatr Psychiatry 2001;16:816-821.

45. Schoenmakers B, Buntinx F, DeLepeleire J. Supporting the dementia family caregiver: the effect of home care intervention on general wellbeing. Aging Ment Health 2010;14:44-56.

46. Burdz MP, Eaton WO Bond JB Jr. Effect of respite care on dementia and nondementia patients an,d their caregivers. Psychol Aging 1988;3:38-42. 